\title{
INSTAURACIÓN DE LA FORMACIÓN Ambiental EN LA UNIVERSIDAD Colombiana
}

\author{
Por: Morelia Pabón Patiño
}

\section{RESUMEN}

Este artículo presenta desde una perspectiva histórica el proceso de instauración del proyecto educativo ambiental en la universidad colombiana, a partir de los años setenta del Siglo XX. El contexto universitario durante la época estuvo determinado por transiciones en la política estatal, las reformas introducidas por la Ley 80 de 1980, y la Ley 30 de 1992, darían lugar a una diversificación de las instituciones universitarias y establecimiento de ciclos (técnico, tecnológico y profesional) en la educación superior. En el sistema se advierte un incremento de instituciones y matricula del sector privado frente a la universidad Estatal, y una nueva distribución geográfica con amplia presencia regional. En este marco se instauraría la formación ambiental en la educación superior.

\section{Elementos de Apoyo en el Surgimiento de los Estudios Ambientales en Colombia}

a educación ambiental tanto a nivel internacional como nacional se considero como parte integrante de la formación profesional y disciplinaria impartida en las universidades, asignándole un importante papel en la transformación cultural frente a la relación naturaleza y sociedad.

La instauración del proyecto educativo ambiental en la universidad colombiana, a partir de los años setenta del Siglo XX. El contexto universitario durante la época estuvo determinado por transiciones en la política estatal, las reformas introducidas por la Ley 80 de 1980, y la Ley 30 de 1992, darían lugar a una diversificación de las instituciones universitarias y establecimiento de ciclos (técnico, tecnológico y profesional) en la educación superior. En el sistema se advierte un incremento de instituciones y matricula del sector privado frente a la universidad Estatal, y una nueva distribución geográfica con amplia presencia regional. En este marco se instauraría la formación ambiental en la educación superior. 
La educación ambiental tanto a nivel internacional y nacional se considero como parte integrante de la formación profesional y disciplinaria impartida en las universidades, asignándole un importante papel en la transformación cultural frente a la relación naturaleza y sociedad. La instauración de la formación ambiental en la universidad colombiana contó con el acompañamiento de la Red de Formación Ambiental Latinoamericana y Nacional y de algunas instituciones rectoras del sistema universitario.

La recepción de ella discurre en los años setenta a través de estudios de ecología, introducidos por grupos académicos generalmente pertenecientes a departamentos o facultades de biología o ecología, comúnmente los estudios fueron incorporados a programas de formación profesional, en los años ochenta la instauración transcurrió simultáneamente con la constitución de núcleos académicos integrados a prácticas interdisciplinarias en torno al estudio de los problemas ambientales nacionales o locales. La educación ambiental se instauro a través de diversas modalidades y proyectos, apertura de programas curriculares de pregrado y postgrado, introducción de cursos de contexto o cátedras ambientales en programas tradicionales, prácticas pedagógicas activas en los contextos locales, conferencias, seminarios y publicaciones específicas.

Al finalizar el decenio del noventa fue evidente el incremento de instituciones y programas de formación ambiental. Algunas instituciones configuraron el campo de estudios ambientales a partir de la constitución de núcleos académicos interdisciplinarios, la actividad académica en este campo generalmente integró las funciones de formación, investigación y proyección social en materia ambiental hecho que suscito algunas modificaciones tanto en el orden académico como en la estructura académica- administrativa, dando lugar al surgimiento de unidades académicas interdisciplinarias como Centros e Institutos, los cuales coexistieron con las tradicionales facultades o departamentos en este quehacer.

\section{El Contexto Universitario Colombiano: A Finales del Siglo XX}

La instauración de la educación ambiental en la universidad colombiana durante el último tercio del Siglo XX afirma la vigencia de la correspondencia entre la dinámica social con el origen y la transformación de la universidad. Atendiendo el carácter de institución educativa, la sociedad asigna a la universidad la función de formar a las jóvenes generaciones en atención a un determinado orden social, en un momento histórico. Esta función de la educación explicada por los teóricos de la reproducción, ${ }^{1}$ fundamenta la comprensión del proceso de instauración del proyecto educativo ambiental, como afirmación a una demanda de la 
sociedad contemporánea en sus dimensiones cognitiva y regulativa, en la formación disciplinar y profesional impartida por la universidad colombiana. ${ }^{2}$

En el panorama de la segunda mitad del Siglo XX, el proceso de modernización productiva suscitó una transformación de la universidad orientada a adaptar la educación superior a los nuevos requerimientos económicos y sociales derivados de la adopción del modelo de "desarrollo hacia adentro" promovido por la CEPAL, basado en el proteccionismo industrial, la sustitución de importaciones, la explotación de los recursos naturales y endeudamiento externo, ${ }^{3}$ este modelo económico origino la política de ampliación en la matricula de la educación superior. En el período finisecular, la política universitaria se adecuaría a las demandas de la apertura económica, y la globalización, consecuentemente el influjo de políticas neoliberales introduce cambios en la concepción de la función del Estado frente a la educación, la tensión entre la concepción de la educación como derecho o mercancía afectaría la financiación y crecimiento de la universidad estatal. Asimismo las reformas de los años ochenta y noventa generarían una diversificación de instituciones y niveles de educación post secundaria. De otra parte, la política educativa del Estado refrendo acuerdos vinculantes establecidos por la comunidad internacional con respecto a nuevas dimensiones educativas urgidas en el contexto contemporáneo, incluido el ideal de formación ambiental que emerge ante el debate y reflexión social sobre el impacto destructor sobre la naturaleza derivado de los modelos productivos.

En los años sesenta el Estado introduce nuevas reformas al sistema de educación superior. La universidad vive una fase de debate y movilización estudiantil y profesoral, exteriorizando la tensión entre la aspiración de autonomía y cogobierno de la comunidad universitaria, y la actitud del ejecutivo de ejercer control y centralización en la toma de decisiones, situación aunada a los recurrentes problemas de financiación. En el contexto de la reforma propuesta conocida como el Plan Básico de Educación Superior, se plantea como uno de sus ejes la estructura interna de la universidad, ${ }^{4}$ basada en el modelo de la universidad norteamericana sugerido en el "Informe Atcon" en 1963. Algunas universidades introducen en su estructura orgánica la figura del "departamento", bajo el cual gravitaría el cumplimiento de las funciones académicas sustantivas de docencia, investigación y la proyección social. ${ }^{5}$ La organización por departamentos reafirmaría el carácter disciplinario por el cual transitaría la actividad académica en la mayoría de las universidades del país durante el último tercio del Siglo XX, en gran medida este modelo de estructura configuraría un escollo para el trabajo interdisciplinario demandado por la instauración de los estudios ambientales. 
Asimismo, la educación superior colombiana durante el último tercio del Siglo XX evidencia una propensión al incremento de instituciones y diversificación de las modalidades educativas. El Decreto Ley 080 de 1980 estableció cuatro modalidades de educación post-secundaria (intermedia profesional, tecnológica, universitaria y avanzada o de postgrado); la Ley 30 de 1992, define a las instituciones de educación postsecundaria, como Instituciones Técnicas, Tecnológicas, Universitarias y Universidades. Al concluir la década del noventa, el número de instituciones universitarias se había cuadriplicado con relación a la cifra existente en el año 1968, y las privadas duplicaban la cifra de las instituciones públicas de educación superior.

Tabla 1

Instituciones Universitarias en Colombia, 1968-1999

\begin{tabular}{|l|l|l|l|l|}
\hline TIPO DE INSTITUCIÓN & $\mathbf{1 9 6 8}(\mathbf{1})$ & $\mathbf{1 9 8 0}(\mathbf{2})$ & $\mathbf{1 9 9 2}(\mathbf{3})$ & $\mathbf{1 9 9 9}(\mathbf{4})$ \\
\hline INSTITUCIONES & & & & \\
TÉCNICAS & & & 54 & 49 \\
TECNOLÓGICAS & & & 58 & 54 \\
UNIVERSITARIAS & 38 & & 68 & 65 \\
\hline UNIVERSIDADES & 17 & & 79 & 93 \\
ESTATALES & 21 & & 46 & 38 \\
PRIVADAS & 24 & & & 55 \\
\hline INSTITUTOS & 14 & & & \\
PÚBLICOS & 10 & & & \\
PRIVADOS & 62 & 188 & 259 & 274 \\
\hline TOTAL INSTITU CIONES & 31 & 132 & 181 & 187 \\
\hline PRIVADAS & 31 & 56 & 78 & 85 \\
ESTATALES & & & & \\
& & & & \\
\hline
\end{tabular}

Fuente: (1) RAMA, Germán, El Sistema Universitario Colombiano, Bogotá, 1973, cuadro 18, p.42 (2), (3), (4) ICFES. Estadísticas de la Educación Superior, 1992-1999

Entre 1960 a 1999 el sistema universitario afirmaría una estructura dual integrada por los sectores públicos y privado, en el cual se observa un crecimiento considerable en la matricula en la educación superior, observada en la tabla 2, las estadísticas permiten inferir desde el primer semestre académico de 1974, la preeminencia de matricula del sector privado frente a la universidad estatal, asociada con el reducido crecimiento en la oferta de cupos del sector público asociada a las restricciones en la financiación estatal de la educación superior. En el decenio del noventa, la concepción de la educación como derecho transita hacía la educación como servicio educativo (incorporado a la noción de mercado), enfoque introducido en la política de Apertura Educativa. ${ }^{6}$ Asimismo a finales del siglo XX la preeminencia del modelo económico neoliberal incremento el proceso de empobrecimiento de la población, ocasionando inaccesibilidad y deserción para sectores de escasos recursos. 
Tabla 2

Colombia Alumnos Matriculados en la Educación Superior según la Naturaleza de las Instituciones, 1960-1999

\begin{tabular}{|l|l|l|l|}
\hline AÑO & TOTAL & OFICIAL & PRIVADO \\
\hline 1960 & 23.013 & 13.639 & 9.374 \\
\hline 1964 & 36.617 & 21.302 & 15.315 \\
\hline 1973 & 124.236 & 62.161 & 62.075 \\
\hline 1974 I SEM & 148.021 & 72.243 & 75.778 \\
\hline 1983 I SEM & 356.000 & 140.963 & 215.037 \\
\hline 1999 I SEM & 877.944 & 294.398 & 583.546 \\
\hline 1999 II SEM & 877.420 & 299.450 & 578.970 \\
\hline
\end{tabular}

Fuente: ICFES. Estadísticas de la Educación Superior

Igualmente, el incremento de instituciones introdujo un nuevo patrón de distribución geográfica de la educación superior, que supera la tendencia de concentración en la capital o ciudades señaladas como los nodos regionales, dominante hasta mediados del Siglo XX. La Ley 30 de 1992, determinó crear un Sistema Regional de la Educación Superior, complementada con la integración regional universitaria dando lugar al establecimiento de los Comités Regionales de Educación Superior CRES entes de coordinación a nivel regional. Asimismo, introdujo el funcionamiento del Sistema de Universidad Estatal, SUE.

La expansión de la matricula universitaria durante este período respondió en gran parte a dinámicas económica, social y demográfica. Efectivamente se presenta una sincronía de tendencias, aumento de la demanda de educación superior por sectores medios y populares, igualmente la dinámica demográfica presento entre 1964 a 1993 un incremento absoluto de la población en edad escolar, incluido el grupo etáreo de educación superior (18 a 24 años), que duplico su tamaño en este lapso de tiempo.

Tabla 3

Población Escolar de Colombia por Grupos de Edad, 1964- 1993

\begin{tabular}{|l|lc|lc|lc|}
\hline GRUPOS DE & \multicolumn{4}{|l|}{$\begin{array}{l}\text { 1964 } \\
\text { EDAD }\end{array}$} & No. & \multicolumn{2}{|l|}{$\%$} & No. & $\%$ & No. & $\%$ \\
\hline $5-6$ & 1.163 .876 & 6.7 & 1.696 .260 & 5.2 & 1.751 .653 & 4.7 \\
\hline $7-11$ & 2.578 .407 & 14.7 & 3.844 .206 & 11.8 & 4.278 .032 & 11.4 \\
\hline $12-17$ & 2.417 .500 & 13.8 & 4.533 .108 & 14.0 & 4.873 .139 & 13.0 \\
\hline $18-24$ & 2.093 .414 & 12.0 & 4.981 .545 & 15.3 & 5.211 .221 & 14.0 \\
\hline TOTAL & 17.484 .508 & 100 & 32.495 .400 & 100 & 37.428 .105 & 100 \\
\hline
\end{tabular}

Fuente: DANE, Censos de Población, 1964, 1985, 1993 
La ampliación en la tasa de cobertura de la educación superior del país, inició un proceso hacia la masificación como se advierte a finales de los años noventa, ${ }^{7}$ especialmente relacionada con la oferta creciente del sector privado.

Tabla 4

Colombia Cobertura de Educación Superior para la Población entre 18 - 24 Años, 1993-1997

\begin{tabular}{|l|l|l|}
\hline AÑO Y ZONA & TASA BRUTA & TASA NETA \\
\hline $1993(1)$ & 15.7 & 8.1 \\
\hline $1997(1)$ & 24.0 & 14.6 \\
\hline
\end{tabular}

Fuente: (1) UNIVERSIDAD NACIONAL DE COLOMBIA (2001) Estadísticas e Indicadores de la Universidad Nacional de Colombia, 2000, Revista de la Oficina de Planeación, No. 4, Cuadro 4, p 135

En este marco de transformaciones fue instaurada la educación ambiental en la formación universitaria como uno de los fines de la educación superior, acogiendo los lineamientos determinados por la Ley 30 para la creación de programas curriculares de educación superior. ${ }^{8}$

Recepción e Instauración de la Formación Ambiental: Itinerario 1972-1999. La formación ambiental orientada hacia la adquisición de conocimientos y apropiación de actitudes y valores comprometidos con la problemática ambiental, asumió diversas modalidades de enfoques y prácticas educativas en las instituciones universitarias, siendo estructurados programas curriculares de pregrado con énfasis en la temática ambiental en el marco de campos profesionales y disciplinares tradicionales; simultáneamente fueron integradas cátedras o asignaturas a los planes de estudio, y algunas instituciones realizan seminarios y prácticas educativas relativos a esta formación.

Recepción en los años setenta: Inicialmente, la fase de recepción correspondió a una tendencia de educación ecológica, motivada entre otros factores, por la orientación oficial contenida en el Código Nacional de Recursos Naturales de 1974, y por el interés de académicos y los grupos ecológicos comprometidos con la ingreso del componente cognitivo y actitudinal del ideal de formación ambiental en el contexto universitario, siendo relevante el papel cumplido en aquella época, por algunos docentes orientados a la instauración de estudios de ecología.

En 1970 la junta directiva de COLCIENCIAS, instauró el grupo de "Desarrollo Tecnológico, Cultura Y Estudios Prospectivos", conocido como el grupo 2000, cuyo objeto de trabajo estuvo ligado a la búsqueda de alternativas de desarrollo y definición de un "Programa Especial de Investigaciones sobre Aspectos 
Ecológicos y Ambientales", con este propósito organizo varios comités, uno de ellos, el "Comité Nacional Hombre y Biosfera" instalado el 30 de Julio de 1971, para actuar en asuntos ecológicos, ${ }^{9}$ la actividad de este comité involucro e influyo el quehacer universitario.

Al ser creado en 1973, el "Consejo de Población y Medio Ambiente", como organismo asesor adscrito al Departamento Nacional de Planeación, con el propósito de: 1- Elaborar y proponer políticas y medidas en materia de población, formación y utilización de los recursos humanos en el país; 2-Fomentar la formación de profesionales y científicos en los campos relativos a población, recursos naturales y medio ambiente, en todos los campos de la educación y la investigación; 3- Promover y recomendar en los distintos niveles de la educación actividades docentes que permitan a los miembros de la sociedad adquirir y practicar los conocimientos sobre los aspectos poblacionales, los recursos naturales y del medio ambiente, 4- Promover y recomendar alguna técnica y financiación a entidades o personas públicas o privadas para desarrollar programas investigativos, docentes y de servicios en los asuntos de Población, recursos naturales y medio ambiente. ${ }^{10}$ Para el logro de estos propósitos el Consejo convoco la acción de la universidad

La legislación estatal formulada en los años setenta, bajo la influencia del Congreso de Estocolmo, comprometió a la universidad colombiana a instaurar la educación ambiental en el proceso de formación universitaria. ${ }^{11}$ A partir de la reglamentación del Código de Recursos Naturales fue expedido el Decreto 1377 de 1978 que faculta al ICFES para brindar apoyo a la institución universitaria en la labor de la sensibilización e información ambiental, mediante la organización de seminarios sobre ecología, preservación ambiental y recursos naturales renovables. La perspectiva de la norma omite la naturaleza interdisciplinaria de la formación ambiental y la supedita al contexto específico de una disciplina, reduciéndola al estudio de la ecología. Igualmente, el Decreto citado determino la asesoría del INDERENA, a las universidades, para la elaboración de monografías y tesis de grado relacionadas con recursos naturales renovables, ecología y protección ambiental». ${ }^{12}$ Asimismo, en lo relativo a la definición de los problemas a investigar, dispuso: «A través de la comisión asesora, el Instituto Nacional de los Recursos Naturales Renovables y del Ambiente presentará periódicamente a las universidades una lista de temas prioritarios que a su juicio requieran ser investigados con el fin de promover el desarrollo de investigaciones interdisciplinarias y, si es posible, ínter universitarias. Para el desarrollo de estas investigaciones el Instituto Nacional de los Recursos Naturales Renovables y del Ambiente suministrará la información necesaria». ${ }^{13}$ 
Lamentablemente en la Comisión Asesora, no existía representación de la comunidad académica o de las instituciones universitarias.

La recepción del proyecto educativo ambiental en la universidad avanzo comúnmente a través de la inclusión de algunas asignaturas como la ecología y el manejo o conservación de los recursos naturales, respondiendo al paradigma vigente a la fecha. En este contexto el ICFES a través de su comité de Ecología, realizo en 1974, el encuentro "Contribución de la Universidad al Estudio y Solución de los Problemas Ecológicos", con la participación de los rectores de las instituciones de educación superior, ${ }^{14}$ de la asistencia a este evento se derivaría la gestión de algunos directivos universitarios por incorporar este ideal de formación en las instituciones de educación superior. A finales del mismo año se realizo el "Primer Seminario Nacional sobre Enseñanza de la Ecología”, de donde surgió el Comité Nacional de Ecología. El ICFES, por medio de este comité, trabajo sobre las

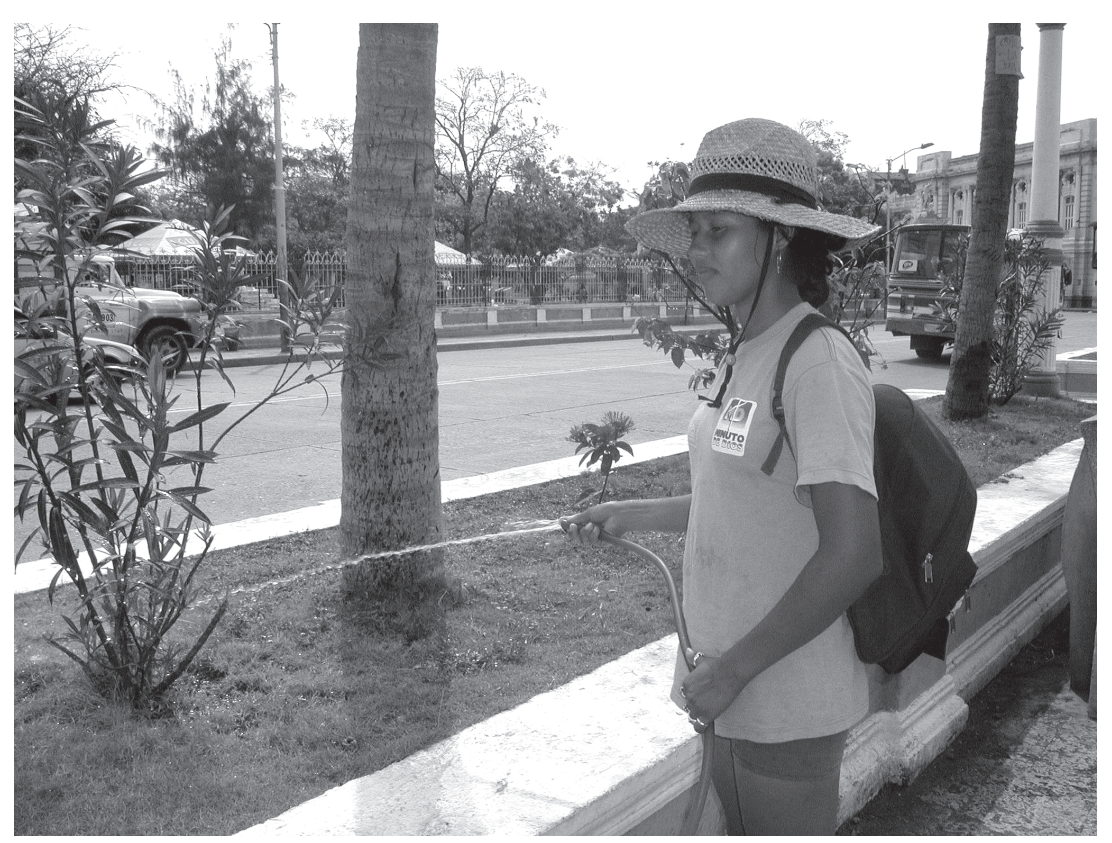

Foto: Mario Lorduy. Sección Publicaciones. Universidad de Cartagena.
Unidades Académicas de ecología para programas universitarios. En octubre de 1975, en la sede de la Universidad Tecnológica de los Llanos Orientales se celebra el "Segundo Seminario Nacional sobre la Enseñanza de la Ecología", abordando cinco temáticas alusivas a: 1 Enseñanza de la ecología en primaria y secundaria; 2Enseñanza de la ecología en la Universidad; 3- Formación de docentes; 4- Investigación científica de la ecología; 5Organización y financiación de las estaciones ecológicas. El seminario recomendó modificar el Comité Nacional de Ecología y crear la Comisión permanente por la defensa de los recursos naturales y el medio ambiente, con sede en la Universidad Católica. Asimismo se acogió la propuesta de organizar una Estación Ecológica en la Universidad Tecnológica de los Llanos (municipio de Restrepo, departamento del Meta), para trabajar como consorcio universitario en el desarrollo de la investigación científica y la formación de docentes universitarios, con participación de investigadores internacionales, y crear una junta directiva con funciones pertinentes al propósito. ${ }^{15}$ Estas propuestas no lograron concretarse. Los tópicos abordados 
en el Seminario evidencian el énfasis en el conocimiento de la ecología como fundamento de esta formación durante el decenio de los setenta. Posteriormente en Octubre de 1977 se realizo en la Universidad Tecnológica de Pereira, el "Tercer Seminario sobre la Enseñanza de la Ecología", las conclusiones del evento se orientaron a: la creación de centros ecológicos; creación de comités regionales; apertura de cursos obligatorios de ecología; la definición de responsabilidades de la universidad; determinación de un marco para la educación y la investigación; y elaboración de cartillas ecológicas para el proceso educativo.

Según el estudio del Centro Internacional de Formación en Ciencias Ambientales, $\mathrm{CIFCA}^{16}$ puede colegirse la recepción del proyecto educativo ambiental en algunas universidades colombianas, al concluir los años setenta. El estudio precisa la introducción de esta tendencia formativa, en programas profesionales de diversas áreas, e igualmente en la formación avanzada. Efectivamente, universidades públicas y privadas habían incluido en los planes curriculares temáticas o asignaturas orientados a incorporar la educación ambiental.

En el contexto de la universidad estatal, se relaciona el emprendimiento de esta formación en la Universidad Nacional sede Bogotá, en la facultad de Ciencias la cual realizaba cursos a nivel de pregrado sobre problemas ambientales, ecología y conservación de recursos naturales; la facultad de ingeniería, llevaba a cabo cursos a nivel de postgrado en ingeniería ambiental y sanitaria; en la facultad de Artes, el departamento de Planificación y Medio ambiente, implemento en el pregrado, estudios sobre Planificación y medio ambiente y asentamientos humanos, al igual que en el postgrado sobre planeación urbana. De otra parte la Universidad Pedagógica Nacional, en programas de formación de docentes, el departamento de Biología efectuaba cursos sobre Ecosistemas y Ecología aplicada; el departamento de Sociales ofrecía estudios sobre Equilibrio Ecológico, Reservas Naturales y Parques Nacionales.

De igual manera, la Universidad del Atlántico integró en los estudios de pregrado del Departamento de Ingeniería Química cátedra de Contaminación del medio ambiente acuático, en la facultad de Arquitectura asignaturas sobre Planeación y urbanismo. La Universidad de Magdalena, implemento en la Facultad de Economía Agrícola, en el Programa de Ecodesarrollo, cursos sobre Ecodesarrollo, Recursos naturales y Problemas agrarios, igualmente en el Programa de Ecología, formación en Conservación de suelos; Contaminación; Ecología y Sistema Natural. A su vez, la Universidad Industrial de Santander, introdujo en los cursos ofrecidos en el pregrado por el departamento de Biología, asignaturas sobre Utilización racional de los suelos, agua, flora y fauna; Equilibrio ecológico y 
Saneamiento Ambiental. La Universidad de Caldas había iniciado la inclusión de asignaturas sobre Manejo y Conservación de Recursos Naturales y Legislación Ambiental, en la facultad de Veterinaria. Asimismo, la facultad de Arquitectura de la Universidad Nacional, sede Manizales, ofrecía cursos de Asentamientos Humanos; Planeación y Diseño regional y urbano. En el campo de la Salud, la Universidad Tecnológica de Pereira, conjuntamente con el INDERENA, efectuó algunos seminarios sobre el tema ambiental, y la introducción de la perspectiva ambiental en el programa de medicina comunitaria.

La universidad privada igualmente incorporo la dimensión cognitiva ambiental en los procesos de formación. La Universidad de los Andes, en el campo de la ingeniería civil introdujo estudios físicos, químicos y ecológicos del manejo de los recursos naturales para estudiar la disposición de uso de aguas, en la época preparaba el proyecto de formación en ingeniería ambiental. Esta universidad inició en agosto de 1977, el postgrado en Planificación de Desarrollo Regional, conjuntamente con el Instituto de Estudios Sociales de La Haya, para lo cual estructuró una línea en torno a un núcleo común de conocimiento de carácter teórico y técnico para la formación de un planificador regional, con integración del enfoque ambiental. ${ }^{17}$ La Universidad Javeriana incorporó la educación ambiental a través de cursos sobre problemas ambientales, ecología y conservación de recursos naturales. En particular, la facultad de Ciencias ofrecía cursos en Ecología naturalista y Ecología humanista; el departamento de Biología a nivel de postgrado impartía la cátedra de Ecología. A nivel de la formación postgraduada de la Facultad de Estudios Interdisciplinarios incorporó la reflexión y análisis de la temática ambiental en los programas de Estudios Políticos; Economía y Recursos Humanos; Educación; Población y en el postgrado en Planeación Urbana y Regional. Algunos proyectos de investigación incorporaron áreas temáticas relacionadas con la Vivienda, Remodelación urbana; Planes de desarrollo; Ordenación Territorial y Manejo Ambiental. En la Universidad Jorge Tadeo Lozano, los programas de pregrado de la Facultad de Agrología incorporan cátedras de conservación y recuperación de suelos y agua; la Facultad de Ciencias del Mar realizó la inclusión de asignaturas relacionadas con Biología Marina y Ecología.

De conformidad con estos hechos es posible conjeturar la recepción del proyecto educativo ambiental en un sector de la universidad colombiana al finalizar los años setenta, alrededor de una docena de universidades habían instaurado esta dimensión educativa comúnmente con la oferta de cursos de ecología, o asignaturas con temáticas ambientales, conservación y manejo de los recursos naturales, las cuales fueron incorporadas a programas de formación tradicional 
a nivel de pregrado. Asimismo se observa el funcionamiento de algunos postgrados con objetos de formación específicos en problemas o estudios ambientales. En esta educación fue relevante la articulación entre teoría y práctica; el carácter interdisciplinario de los estudios ambientales introdujo una relación entre diversas comunidades disciplinarias, demandando ruptura en el aislamiento tradicional o compartimentación de las facultades y departamentos. ${ }^{18}$ En forma general, en la fase de recepción en los años setenta impera un acercamiento al conocimiento de los ecosistemas y de temáticas de estudios ambientales, la acción educativa actitudinal se sintetiza en la sensibilización de la comunidad educativa frente a los problemas del entorno, el elemento cognoscitivo se asimilo al estudio de los ecosistemas.

La instauración de la formación ambiental en el decenio del ochenta se ve fortalecida con la realización de dos eventos

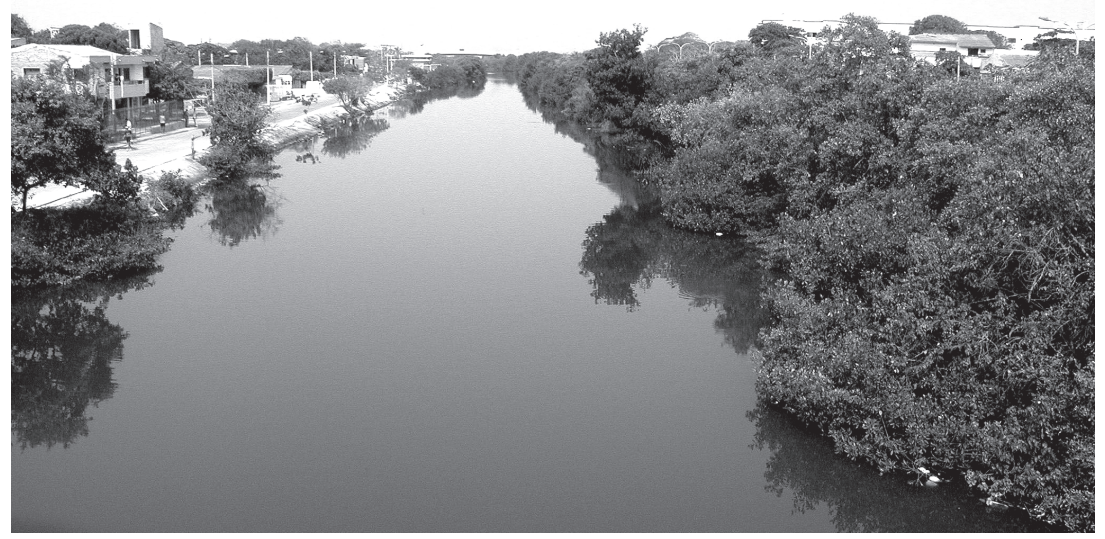

Foto: Mario Lorduy. Sección Publicaciones. Universidad de Cartagena. académicos. En 1985 se llevo acabo el primer Seminario Sobre Universidad y Medio Ambiente para América Latina y el Caribe", y el "Primer Seminario Nacional Sobre Universidad y Medio Ambiente" efectuado durante los días 27, 28 y 29 de abril de 1988, en las dependencias de la Universidad Nacional de Bogotá, dando cumplimiento a una de las recomendaciones emanadas del Primer Seminario Latinoamericano. La celebración del Primer Seminario Nacional estuvo mediada por la coordinación interinstitucional del ICFES, la Red Nacional de Formación Ambiental y la Universidad Nacional. Este evento constituye un acontecimiento fundacional del proceso de instauración de la educación ambiental, allí se formularon lineamientos para el desarrollo de una política académica ambiental en la universidad colombiana, las cuales fueron consignadas en la "Declaración de Bogotá, Orientaciones Para la Política Ambiental en la Universidad Colombiana". El objetivo general del Primer Seminario Nacional estuvo encauzado a la formulación de políticas, estrategias y mecanismos operativos para la incorporación de la dimensión ambiental en los estudios superiores del país, abordando temas relacionados con: a- La política ambiental universitaria; bIncorporación de la dimensión ambiental en las carreras existentes y establecimiento de políticas para la creación de carreras ambientales; cInvestigación ambiental; d- Programas de extensión; e- Interdisciplinariedad; 
f- Elaboración de un plan de acción. Durante la sesión inaugural del Seminario Nacional, El ICFES presento la ponencia titulada: "Importancia de la Incorporación de la Dimensión Ambiental en La Educación Superior"; asimismo fueron expuestas las "Conclusiones del Seminario Sobre Universidad y Medio Ambiente para América Latina y el Caribe", y el "Diagnóstico sobre Programas Ambientales Universitarios" en Colombia elaborado por Augusto Ángel Maya.

El seminario puntualizó como objetivos: 1- Definir orientaciones de política académica y administrativa para la incorporación de la dimensión ambiental en los estudios superiores. 2- Hacer sugerencias sobre el desarrollo de programas de pregrado y postgrado en temas ambientales. 3- Sugerir estrategias y mecanismos para incorporar la dimensión ambiental en las carreras universitarias existentes. 4- Establecer orientaciones y prioridades de investigación ecológicas y ambientales sobre los problemas de más urgente solución. 5- Sugerir estrategias que permitan incrementar la capacitación del personal docente y el contacto con la comunidad, tales como cursos de extensión, publicaciones, revistas, asesorías ambientales, etc. 6- Diseñar políticas y estrategias para reforzar el desarrollo de estudios interdisciplinarios tanto en el campo investigativo como en la docencia. 7- Establecer mecanismos de organización que permitan llevar a la práctica las conclusiones del Seminario Latinoamericano y del presente seminario". ${ }^{19}$

Los participantes convocan a fortalecer esfuerzos para asegurar la incorporación del proceso de formación ambiental, y desarrollar propuestas investigativas entre diversas facultades o unidades académicas atendiendo la naturaleza interdisciplinaria de los estudios ambientales. En este sentido, en primer lugar se reconoció la insuficiencia de las acciones implementadas en la Universidad para responder al reto de los problemas ambientales declarando en consecuencia la necesidad de "formular políticas y estrategias que permitieran el desarrollo de un plan de acción a nivel nacional, regional y local para intensificar la incorporación de la dimensión ambiental en los estudios superiores". Los asistentes reclamaron del apoyo estatal e institucional del sector para la planeación y gestión del proceso, afirmando: «Para fortalecer el esfuerzo de las distintas universidades es indispensable que los organismos que orientan la política universitaria e investigativa cooperen con el diseño y en la ejecución de un plan de acción nacional para incrementar la conciencia ambiental en el ambiente universitario». ${ }^{20}$ De otra parte, el seminario recomendaría la creación de unidades académicas interdisciplinarias propiciando rupturas en la tradicional organización académica, "Para lograr un ambiente de trabajo interdisciplinario al interior de las universidades es indispensable propiciar la creación de espacios 
académicos que lo permitan. Para ello se sugiere la creación de centros, áreas u otras formas académicas que cuenten con los recursos suficientes, con el nivel de articulación necesario para desarrollar docencia e investigación interdisciplinaria. Igualmente es indispensable realizar un mayor esfuerzo para ampliar la conciencia ambiental a través de programas de extensión tanto al interior del medio universitario como en los espacios de la opinión pública». En 1988 se habían constituido, el Centro de Investigaciones Ambientales de la Universidad de Antioquia; el Centro Ecológico Integrado de Manizales, CEIMA; el Centro de Estudios Ecológicos de la Universidad de Córdoba, el Centro de Estudios Interdisciplinario, CIDER, de la Universidad de los Andes; el Centro de Estudios Geográficos de la Universidad del Cauca; el Centro de Acción Ambiental de la Universidad del Valle, y el Centro Ecológico de la Provincia de Pamplona, en la Universidad de Pamplona. La constitución de estos centros, se fundamentó en los nichos académicos existentes en instituciones con alguna tradición en el trabajo interdisciplinario sobre problemas que incorporan el estudio de la relación entre los sistemas biofísico y social.

Un análisis sobre alcance de los propósitos trazados en el Primer Seminario Nacional sobre universidad y medio ambiente, fue expresado por Augusto Ángel, quien considera: "No podía esperarse que un seminario abriese definitivamente el camino o que unas cuantas conclusiones fuesen suficientes para que la universidad comprendiese la importancia de la problemática ambiental y afrontase el reto de la interdisciplina." Desde esta perspectiva se conjetura el carácter limitado de los eventos académicos como promotores para la recepción e inserción de proyectos de formación, y configuración de campos de conocimiento, ante lo cual se formula un llamado a reconocer otros factores implícitos en el proceso de generar, transmitir y aplicar conocimiento en la universidad, comprometiendo no sólo la constitución de núcleos de labor académica, o comunidades académicas sino igualmente la gestión académicoadministrativa. Los asistentes precisamente no fueron rectores o decanos, fueron algunos académicos abanderados e instauradores de la visión ambiental en el medio universitario, pero que por lo general no ejercían cargos en la administración académica. Precisamente Ángel cuestiono sobre ícuál fue el efecto que tuvo el Seminario en las universidades que enviaron representantes?, o, ique resonancia o divulgación tuvo en las universidades que no asistieron? ${ }^{21}$

El balance sobre la inclusión de la educación ambiental en la formación universitaria en $1988,{ }^{22}$ verifica la existencia de veinticinco programas de pregrado y postgrado. En forma general ellos asumían como objeto de estudio la relación entre el sistema social y los ecosistemas, articulando las ciencias 
naturales y sociales. El estudio diagnóstico de Augusto Ángel sobre la formación ambiental identifico veinticinco programas curriculares con predominio de catorce programas en ingeniería, cinco en las ciencias naturales, y dos en áreas administrativa e interdisciplinaria respectivamente. Igualmente destaca la presencia hegemónica de la ecología en el momento de recepción, sobre lo cual afirma: «la perspectiva ambiental está siendo atrapada por el reduccionismo propio de la formación universitaria. Los ecólogos la adoptan como propia y lo mismo lo hacen los ingenieros. Las Ciencias Sociales todavía no han empezado a modificar sus perspectivas para entrar en el análisis de la problemática ambiental como crisis de la civilización $»^{23}$. Igualmente establece como horizonte conceptual de la formación ambiental:

El análisis de la relación entre el sistema social y la base ecosistémica. Por consiguiente, presenta una interdisciplinariedad, articulando metodológica y funcionalmente tanto las ciencias naturales como sociales, el plan de estudios de una carrera de formación ambiental organiza un programa que comprende el funcionamiento del ecosistema natural, y la forma como este es artificializado por la actividad humana a través de la plataforma tecnológica y la manera como la organización social y cultural determina dichas transformaciones. ${ }^{24}$

Este estudio acoge esta conceptualización, adicionalmente creemos estos programas constituyen una modalidad de la inserción de la educación ambiental en la educación superior, en la medida que atienden explícitamente el componente cognitivo de la formación ambiental, asimismo en los fines educativos expresados en sus objetivos subyace el componente regulativo o actitudinal en la formación de los estudiantes.

De otra parte, el estudio sobre el "Perfil ambiental de Colombia", al examinar la introducción de la formación ambiental en la universidad colombiana a finales de los años ochenta, reconoció una relativa ausencia de programas en el campo ambiental, y existencia de carreras relacionadas con los recursos naturales y del ambiente. Evidenció la inclusión de la reflexión ambiental, especialmente en el campo de: las ciencias básicas físico biológicas, la química, biología y geología; las ciencias sociales básicas, especialmente en la antropología, y en las- ciencias sociales aplicadas a nivel del planeamiento urbano regional; en ciencias trandisciplinarias como la ecología, la geografía y un poco la historia; en las ciencias naturales aplicadas, como la ingeniería civil y sanitaria, ingeniería de recursos bióticos (agraria, forestal y pesquera), ingeniería de recursos abióticos (minería y la hidráulica); y en las ciencias de la salud. ${ }^{25}$ 
Tabla 5

Colombia Programas Ambientales según Niveles de Formación

y Area de Conocimiento, 1988

\begin{tabular}{|l|c|c|c|c|c|}
\hline \multicolumn{1}{|c|}{$\begin{array}{c}\text { NIVEL } \\
\text { AREA }\end{array}$} & MAESTRÍA & ESPECIALIZACIÓN & PROFESIONAL & TECNOLÓGICO & TOTAL \\
\hline Ingenierías & 6 & 1 & 4 & 3 & 14 \\
\hline Educación & & & 1 & & 2 \\
\hline Administración & 1 & & 1 & & 5 \\
\hline $\begin{array}{l}\text { Ciencias naturales } \\
\text { Ecología }\end{array}$ & 2 & & & & 2 \\
\hline Ciencias sociales & & & 6 & 5 & 24 \\
\hline $\begin{array}{l}\text { Estudios } \\
\text { Interdisciplinarios }\end{array}$ & 2 & 1 & 6 & & 2 \\
\hline TOTAL & 12 & 1 & & & \\
\hline
\end{tabular}

Fuente: ICFES - INDERENA, Ángel Augusto (1989) Diagnóstico Nacional sobre Programas Ambientales Universitarios. p 5

Para el año 1989 se observa la incorporación de la educación ambiental en 18 universidades del país y apertura de treinta y seis (36) programas de formación ambiental. ${ }^{26}$ Existían 16 programas de ingeniería a nivel profesional, especialización y maestría, conexos con la formación ambiental, incluyendo diez programas de ingeniería sanitaria, forestal, geográfica, ambiental o sanitaria y tres especializaciones y tres maestrías en ingeniería ambiental o sanitaria, se asumían estudios ambientales relacionados con la contaminación del aire y agua, seguridad industrial y medio ambiente, manejo de desechos sólidos, diseño de sistemas de control de contaminación del aire y el agua. Adicionalmente a este objeto cognitivo se incluía en los fines educativos un componte actitudinal. En el campo de la ecología y las Ciencias Naturales, el objeto de estudio de los programas era relativo a la estructura y funcionamiento de los ecosistemas. Para finales de los años ochenta, existía un programa de pregrado en Ecología en la Fundación Universitaria de Popayán, y la Especialización en Ecología de la Universidad de Nariño, este programa incorporo como temática, el diseño experimental, métodos estadísticos orientados a la metodología investigativa y a la enseñanza de la ecología, genética y dinámica de poblaciones, estructura y función de los ecosistemas, aspectos científicos en limnología y oceanografía; geografía física, planificación y conservación de los recursos naturales y legislación ambiental, el Taller realizado en mayo de 1987 en esta universidad, sobre la definición de líneas de Investigación apoyado por la Red de Formación Ambiental, estableció como objeto de investigación, los problemas ambientales del mar en la Costa Pacífica del departamento de Nariño; y la Laguna de la Cocha; la problemática ambiental en la ciudad de Pasto; la salud ambiental; la degradación de los suelos frente a la producción. En el área administrativa, se incorpora esta formación en dos programas, uno de ellos a nivel profesional en Administración Sanitaria y Ambiental, en la Universidad Distrital, y la 
Universidad Nacional sede Medellín en la Maestría en "Planeación Urbana". En el campo de las Ciencias Sociales comúnmente se efectúa la reflexión ambiental en los programas de geografía, y antropología, además de la inclusión de asignaturas específicas o temáticas en algunos planes curriculares establecidos. En la década del ochenta afloraron algunos proyectos curriculares en el contexto de las Ciencias Sociales, relacionados con el "planeamiento urbano y regional" en los cuales fue axiomática la inclusión de elementos cognitivos y actitudinales relativos al ambiente. Atendiendo la naturaleza interdisciplinaria de los estudios ambientales, desde los años iniciales de la década existían dos proyectos de maestría con esta característica, en las universidades Javeriana y Andes, la maestría de "Saneamiento y Desarrollo Ambiental" y "Medio Ambiente y Desarrollo Integrado".

\section{Cuadro 1}

Instauración de Formación Ambiental por Nivel e Institución, 1989

\begin{tabular}{|c|c|c|c|}
\hline INSTITUCION & PROFESIONAL & ESPECIALIZACIÓN & MAESTRÍA \\
\hline $\begin{array}{l}\text { Fundación Universitaria } \\
\text { de Popayán }\end{array}$ & 1- Ecología & & \\
\hline Universidad de Nariño & & 21-Ecología & \\
\hline Universidad del Valle & $\begin{array}{l}\text { 2-Ingeniería Sanitaria } \\
\text { 3-Biología }\end{array}$ & & \\
\hline Universidad de Antioquia & $\begin{array}{l}\text { 4-Ingeniería Sanitaria } \\
\text { 5-Biología }\end{array}$ & & $\begin{array}{l}\text { 25-Biología, con énfasis } \\
\text { en Ecología. }\end{array}$ \\
\hline $\begin{array}{l}\text { Universidad Pontificia } \\
\text { Bolivariana }\end{array}$ & & 22-Ingeniería Ambiental & \\
\hline $\begin{array}{l}\text { Universidad Nacional } \\
\text { Medellin }\end{array}$ & 6-Ingeniería Forestal & & 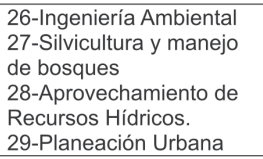 \\
\hline $\begin{array}{l}\text { Universidad Nacional } \\
\text { Bogotá }\end{array}$ & 7-Biología & $\begin{array}{l}\text { 23-Ingeniería Ambiental } \\
\text { 24-Ingeniería sanitaria }\end{array}$ & $\begin{array}{l}\text { 30-Ingeniería Ambiental } \\
\text { 31-Ingeniería Sanitaria }\end{array}$ \\
\hline U. Pedagógica Nacional- & $\begin{array}{l}\text { 8-Licenciatura en } \\
\text { Biología }\end{array}$ & & \\
\hline Universidad Distrital & $\begin{array}{l}\text { 9-Ingeniería Forestal } \\
\text { 10-Administración } \\
\text { Sanitaria y ambiental }\end{array}$ & & \\
\hline Universidad Javeriana & 11-Biología & & $\begin{array}{l}\text { 32-Saneamiento y } \\
\text { Desarrollo Ambiental }\end{array}$ \\
\hline $\begin{array}{l}\text { Universidad Jorge Tadeo } \\
\text { Lozano }\end{array}$ & $\begin{array}{l}\text { 12-Biología Marina } \\
\text { 13-Ingeniería Geográfica }\end{array}$ & & \\
\hline Universidad de los Andes & 14-Biología & & $\begin{array}{l}\text { 33-Biología, área ecología } \\
\text { Animal } \\
\text { 34-Medio Ambiente y } \\
\text { desarrollo }\end{array}$ \\
\hline $\begin{array}{l}\text { Universidad INNCA de } \\
\text { Colombia }\end{array}$ & 15-Ingeniería ecológica & & $\begin{array}{l}\text { 35-Ecología, sanidad } \\
\text { Ambiental Y Medicina } \\
\text { preventiva }\end{array}$ \\
\hline Universidad de la Salle & 16- Ingeniería Sanitaria & & \\
\hline UPTC- IGAC- Bogotá & & & 36-Geografía \\
\hline $\begin{array}{l}\text { Corporación Universitaria } \\
\text { de Boyacá }\end{array}$ & $\begin{array}{l}\text { 17-Ingeniería Ambiental y } \\
\text { Sanitaria }\end{array}$ & & \\
\hline $\begin{array}{l}\text { Fundación Universitaria } \\
\text { de García Rovira }\end{array}$ & 18-Ingeniería Forestal & & \\
\hline Universidad Quindio & $\begin{array}{l}\text { 19-Licenciatura. Biología } \\
\text { y Educación Ambiental }\end{array}$ & & \\
\hline Universidad del Tolima & 20-Ingeniería Forestal & & \\
\hline
\end{tabular}

Fuente: ICFES, SISTEMA DE INFORMACIÓN; ICFES, SUBDIRECCIÓN ACADÉMICA, RED DE FORMACIÓN AMBIENTAL. Programas y actividades ambientales en la educación superior, Documento de trabajo, División de Formación Avanzada, Bogotá, 1990 
Al concluir los años ochenta alrededor de un centenar de programas formación en los diferentes niveles (técnico, tecnológico, pregraduado y postgraduado) incluían la perspectiva educativa ambiental, bajo la modalidad de cátedras ambientales o cátedras de contexto (Universidad la Sabana). No obstante, en términos cuantitativos puede considerarse incipiente la instauración del ideal de formación ambiental, considerando el número de instituciones y programas curriculares. A partir de la información de la subdirección académica del ICFES, en 1989 se puede identificar la inclusión de asignaturas o cátedras relacionadas con el proyecto educativo ambiental, en cerca tres decenas de instituciones de educación superior, a través de setenta programas de formación profesional o disciplinar. Esta cifra permite inferir que a la época solamente quince de cada cien instituciones había introducido por lo menos una cátedra con el componente cognitivo ambiental. La inclusión de la reflexión ambiental comúnmente se presentaba en programas de formación de educadores de ciencias naturales, biología y química, ciencias sociales y geografía, en formación tecnológica química, minera, agropecuaria, obras civiles y geotecnología, y en la formación profesional de agronomía, veterinaria, zootecnia, medicina y programas de administración.

Institucionalización años noventa: Durante los años noventa se registra una a creciente difusión y conciencia sobre los problemas ambientales y la instauración de proceso formativo ambiental en la educación superior.

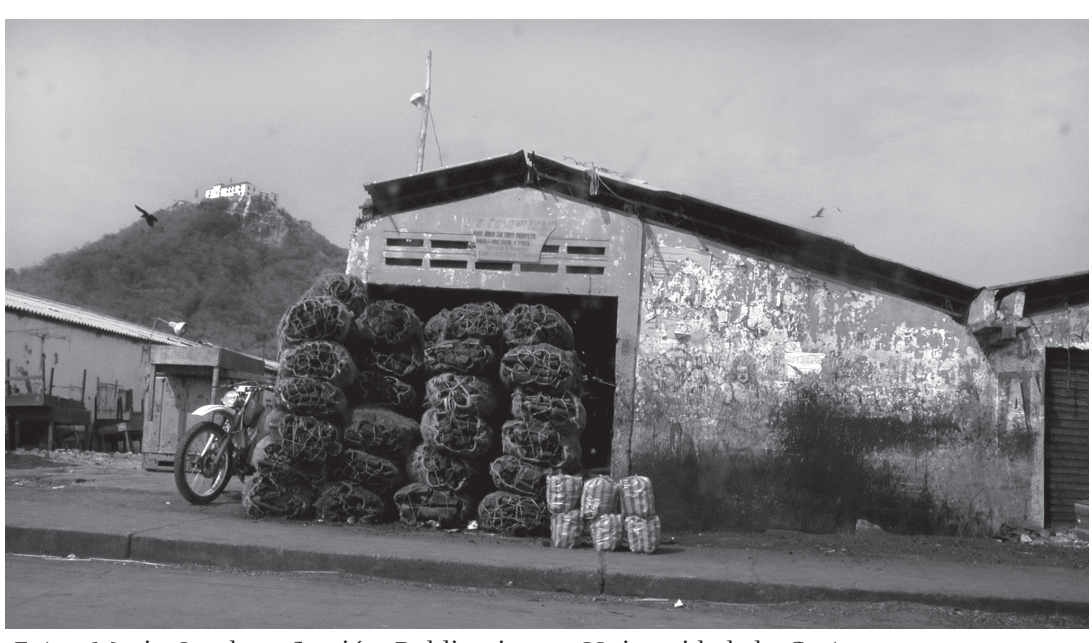

Foto: Mario Lorduy. Sección Publicaciones. Universidad de Cartagena.

Primordialmente, la Carta Constitucional de 1991, oriento la formación del colombiano en el respeto a los derechos humanos, la paz, la democracia; la práctica del trabajo y la recreación para el mejoramiento cultural, científico, tecnológico y para la protección del medio ambiente. Asimismo, la Ley de Educación Superior (Ley 30 de 1992), identifico como uno de sus objetivos: "Promover la preservación de un medio ambiente sano y fomentar la educación y cultura ecológica". La universidad dispuso de autonomía académica para definir proyectos institucionales orientados al impulso de la formación y cultura ambiental en la educación superior, en los marcos establecidos por la Ley. De igual manera, el ascenso de la formación ambiental en la educación superior respondió a requerimientos de algunas misiones estructuradas por el Estado para 
estudiar el fomento del desarrollo científico y tecnológico del país, y de la educación superior, ellas introdujeron demandas relativas a esta formación. Propuestas frente a este propósito fueron realizadas en el contexto del Programa de Ciencias del Medio Ambiente y el Hábitat (1992); la Misión de Ciencia, Educación y Desarrollo (1994), la misión para la Modernización de la Universidad Pública (1994), y la Comisión Nacional para el Desarrollo de la educación Superior (1997). Estas exhortaciones se convierten en factor relevante para la apropiación de la formación ambiental durante el período finisecular en la universidad.

El Sistema Nacional de Ciencia y Tecnología ${ }^{27}$ configurado a partir de la expedición de la Ley de Ciencia y Tecnología, incluyo entre sus programas el de Ciencias del Medio Ambiente y el Hábitat, ${ }^{28}$ en 1992 el Simposio Nacional del Programa definió líneas de investigación, y constitución de redes de investigadores, ${ }^{29}$ aunque allí no se definan líneas o redes en materia educativa ambiental, es posible identificar algunas sugerencias relacionadas con la formación ambiental en la universidad, en el Plan de Investigaciones en Medio Ambiente y Hábitat, PIMAH, formulado por Germán Márquez, que propone un "Programa de Conceptualización y Epistemología Ambiental", con los objetivos de: 1- Explorar el significado de lo ambiental en el contexto de las diferentes disciplinas y como espacio interdisciplinario de reflexión y análisis de la realidad nacional. 2- Desarrollar estrategias de incorporación de la perspectiva ambiental en la formación y el ejercicio profesional de las diversas disciplinas. 3- Crear las condiciones para una formalización de la educación en temas ambientales a nivel profesional y de especialización». ${ }^{30}$ Márquez subrayo la naturaleza interdisciplinaria y de proyección a los problemas del contexto inherente a la formación ambiental impartida en los programas de pregrado y post grado, apoyados en los seminarios nacionales sobre universidad y medio ambiente e involucrando a los institutos o centros de investigación.

El estudio de los problemas ambientales locales permitió iniciar la estructuración de una comunidad académica involucrada en la generación y transmisión de conocimiento ambiental, a la luz de desarrollos académicos endógenos en este campo fue posible durante esta década fortalecer las condiciones para su institucionalización. Asimismo bajo la reflexión realizada durante el Primer Seminario Nacional sobre Universidad y Medio Ambiente, se corroboro la validez de creación de nuevas unidades académico-administrativas para estimular la interdisciplina, generación de conocimiento, y formación ambiental. Durante los años noventa, fueron creados el Instituto de Estudios Ambientales de la Universidad Nacional, IDEA (diciembre de 1991), el IDEADE de la Universidad 
Javeriana (5 de junio de 1991); el Instituto de Investigaciones Ambientales junto con la facultad de Ciencias Ambientales de la Universidad Tecnológica de Pereira, creado junto con la Facultad de Ciencias Ambientales (20 de diciembre de 1991); y el Centro de Estudios Ambientales para el Desarrollo Sostenible CEADES, de la Universidad Autónoma de Occidente. En años anteriores habían sido instaurados el Centro de Investigación Ambiental, CIA de la Universidad de Antioquia, el Centro de Investigación para el Desarrollo Regional, CIDER de la Universidad de los Andes Estos centros e institutos fueron viables gracias a la constitución de comunidades académicas interdisciplinarias, su actividad soporto la institucionalización de campo de estudios ambientales.

El sistema universitario colombiano durante el período finisecular como se señalo preliminarmente observo un incremento de instituciones, estudiantes y programas, en algún grado influida por una visión de mercado, este hecho afecta en algún grado la oferta creciente de programas de formación ambiental. La influencia de esta tendencia puede explicar de algún modo la eclosión de programas relativos al campo ambiental, además del reconociendo al requerimiento social por esta formación. ${ }^{31}$ Los programas de pregrado referidos al objeto de conocimiento ambiental alcanzan en el año 1999, un número de $190,{ }^{32}$ alrededor del 5\% de los programas de educación superior del país. El incremento en un período de 11 años es de casi 8 veces, de alguna manera este ascenso se fundamenta en una mayor estructuración del campo de estudios ambientales en las instituciones universitarias del país.

Tabla 6

Colombia Programas Ambientales según Niveles de Formación

y Campo de Conocimiento, 1999

\begin{tabular}{|l|c|c|c|c|c|c|}
\hline $\begin{array}{l}\text { NIVEL } \\
\text { CAMPO }\end{array}$ & MAESTRÍA & ESPECIALIZACIÓN & PROFESIONAL & TECNOLÓGICO & TÉCNICO & TOTAL \\
\hline Ingenierías & 3 & 13 & 37 & 4 & 1 & 58 \\
\hline Educación & - & 18 & 25 & - & - & 43 \\
\hline Administración & 6 & 35 & 5 & 5 & 3 & 55 \\
\hline $\begin{array}{l}\text { Ciencias } \\
\text { Naturales }\end{array}$ & 1 & 11 & 3 & 4 & - & 19 \\
\hline $\begin{array}{l}\text { Ciencias } \\
\text { Sociales }\end{array}$ & - & 7 & 1 & - & - & 8 \\
\hline $\begin{array}{l}\text { Ciencias } \\
\text { Agrarias }\end{array}$ & 2 & 2 & 1 & 2 & - & 7 \\
\hline TOTAL & 12 & 86 & 72 & 16 & 4 & 190 \\
\hline
\end{tabular}

Fuente: ICFES (1999) SISTEMA DE INFORMACIÓN

Atendiendo las modalidades y niveles de formación, los programas de formación ambiental en 1999, correspondían 4 a la formación técnica; 16 programas de nivel tecnológico, 62 programas profesionales o disciplinares, 86 especializaciones 
y 12 maestrías. El ascenso cuantitativo de programas de formación profesional y disciplinaria a los cuales le fue incorporado un énfasis en los estudios ambientales no logro hacer ruptura con la proverbial estructura de las unidades académicas (facultades, departamentos) y de los planes curriculares, la configuración de estos programas se realizo a partir de introducir énfasis o áreas complementarias de estudios ambientales a la formación disciplinar, y profesional existente en el sistema de educación superior. En general se configuro una heterogénea titulación de programas con enfoque ambiental, al interior de los tradicionales campos de formación profesional universitaria del país, efectivamente la formación ambiental surgió relacionada con programas en las áreas de la ingeniería, la administración y/o gestión, la educación, la química, las ciencias agropecuarias, de la salud, las ciencias naturales y las ciencias sociales, en algunas universidades hizo irrupción la formación disciplinaria en ecología.

La importancia de incorporar la dimensión ambiental en la formación de los educadores fue reconocida tanto en el concierto internacional, como a nivel nacional, para fundamentar la instauración del ideal de formación ambiental en las nuevas generaciones. El Primer Seminario Latinoamericano sobre universidad y medio ambiente señalo la necesidad de actualizar a los profesores en ejercicio de primaria y secundaria en la perspectiva medioambientales, instando a las universidades a brindar este tipo de entrenamiento. ${ }^{33}$ De igual manera, directivas educativas nacionales reconocían la importancia de introducir la educación ambiental en la formación de educadores, el director del ICFES, José Revelo, en el discurso inaugural del Primer Seminario Nacional de Universidad y Medio Ambiente en 1988, afirmaba: "formar educadores dentro de reflexiones teóricas y metodológicas capaces de difundir los valores, crear actitudes y propiciar experiencias formativas de largo alcance que contribuyan a crear una conciencia colectiva en torno a la conservación del medio ambiente como sustento biofísico de la permanencia del hombre". ${ }^{44}$ En 1999 el Sistema de Información del ICFES, registro la existencia de 43 programas en Educación Ambiental, 25 Licenciaturas y 18 Especializaciones. Además se presento la inclusión de asignaturas relacionadas con el conocimiento y formación de actitudes y valores relativos a la relación con el entorno, en licenciatura de diferentes áreas con predominio en la Licenciatura de Educación Ambiental integrada con las Ciencias Naturales. ${ }^{35}$

La dinámica académica en algunas instituciones fue potenciando la constitución de comunidades aplicadas al estudio y resolución de problemas ambientales, este hecho promueve nuevas formas organizativas como los centros o institutos 
de investigación, y la factibilidad de estructurar programas de postgrado a nivel interdisciplinario. Es evidente la apertura de programas referidos a la actualización y perfeccionamiento de conocimientos en materia ambiental, el Sistema de Información del ICFES en 1999 registró 84 programas de especialización ambiental, en diferentes áreas profesionales y disciplinares, con un predominio del área de la Administración con treinta y cinco (35) programas de especialización en Gestión Ambiental, y Recursos Naturales, en el campo de la Educación se registran dieciocho (18), trece (13) correspondían a la Ingeniería, once (11) a las Ciencias Naturales, siete (7) a las Ciencias Sociales, y dos (2) a las Ciencias Agrarias. Los programas de maestría afincados en el desarrollo investigativo, y estructuración de grupos, programas, líneas y proyectos de investigación alcanzan un número reducido, situación común en otras áreas de conocimiento. En 1999 se registran doce programas de maestrías principalmente en universidades estatales o privadas de mayor desarrollo, en las cuales se había presentado un proceso de constitución de comunidades académicas e institucionalización del campo de estudios ambientales.

Cuadro 2

Colombia Programas de Maestrías en el Campo Ambiental: 1999

\begin{tabular}{|c|c|c|}
\hline INSTITUCIÓN & CIUDAD & MAESTRÍAS \\
\hline Universidad del Valle & Cali & 1. Ingeniería Ambiental y Sanitaria \\
\hline $\begin{array}{l}\text { Universidad Pedagógica y } \\
\text { Tecnológica de Colombia. }\end{array}$ & Tunja & 2. Ingeniería Ambiental \\
\hline Universidad de Antioquia & Medellín & 3. Ingeniería Ambiental \\
\hline Universidad Nacional & Medellín & $\begin{array}{l}\text { 4. Bosques y Conservación Ambiental } \\
\text { 5. Estudios Urbano-Regionales }\end{array}$ \\
\hline Universidad Nacional & Bogotá & 6. Ecología \\
\hline Universidad de Los Andes & Bogotá & $\begin{array}{l}\text { 7. Economía del Medio ambiente y } \\
\text { Recursos Naturales }\end{array}$ \\
\hline Universidad Javeriana & Bogotá & $\begin{array}{l}\text { 8. Gestión Ambiental para el Desarrollo } \\
\text { Sostenible } \\
\text { 9. Desarrollo Sostenible de Sistemas } \\
\text { Agrarios }\end{array}$ \\
\hline Universidad Nacional & Manizales & 10. Medio Ambiente Y Desarrollo \\
\hline Universidad de Caldas & Manizales & 11. Agroecología \\
\hline Universidad del Tolima & lbagué & $\begin{array}{l}\text { 12. Planificación y Manejo Ambiental de } \\
\text { cuencas Hidrográficas. }\end{array}$ \\
\hline
\end{tabular}

Fuente: Sistema de Información ICFES. UNIVERSIDAD NACIONAL DE COLOMBIA, OFICINA DE PLANEACIÓN (2000) Estadísticas e Indicadores de la Universidad Nacional de Colombia, Revista de la Oficina Nacional de Planeación, Cuadro 27, p. 184-188.

Los programas de maestría al igual que el pregrado, afirmaron como tendencia el predominio de programas del área administrativa, efectivamente la mitad de ellos (seis) asumían la "Gestión Ambiental". Tres correspondían al área de la ingeniería, particularmente en "Ingeniería Sanitaria y Ambiental". En el área de las Ciencias Naturales, dos programas se encauzaron a la "Ecología", y "Bosques y Conservación Ambiental". Finalmente orientada a la atención de la seguridad 
alimentaría, problema estructural del desarrollo nacional fue objeto de la maestría en "Agroecología".

La institucionalización del proyecto educativo ambiental en la universidad colombiana dispuso del acompañamiento y asesoría de la Red Nacional de Formación Ambiental, constituida en 1981 bajo diferentes entes de coordinación. Entre 1981 a 1985, la coordinación fue realizada por el INDERENA; posteriormente fue desempeñada por el Instituto Colombiano para el Fomento de la Educación Superior, ICFES, designación hecha en 1986, por el Programa de las Naciones Unidas para el Medio Ambiente, PNUMA. Finalmente, a partir de diciembre de 1995 por decisión de la Junta Consultiva de Ministros del PNUMA, la coordinación de la red fue asignada al Ministerio de Medio Ambiente, dando paso simultáneamente a la redefinición de misión y objetivos.

\section{Conclusiones}

El proceso de recepción de la formación ambiental en la educación superior se inicio en los años setenta con la introducción de estudios relacionados particularmente con la ecología; y la conservación de los recursos naturales. Posteriormente, en los años ochenta se inician programas de formación profesional en el campo ambiental y se incorpora la temática en la formación de otras profesiones y disciplinas. En el decenio de los noventa, continuaron vigentes estas modalidades educativas, y aumenta el número de programas curriculares, paralelamente se organizan comunidades y estructuras académicas en el campo de estudios ambientales, avanzó las funciones de investigación y la extensión sobre problemas ambientales locales y nacionales, que fundamentan la apertura de programas de formación postgraduada.

\section{CITAS}

\footnotetext{
${ }^{1}$ Sobre la reproducción y la función simbólica cumplida por el componente regulativo del proyecto educativo nos fundamentamos en los planteamientos de la teoría de la Reproducción expuesta por BOURDIEU Pierre Bourdieu y Jean-Claude Passeron. La reproducción, elementos para una teoría del sistema de enseñanza, Editorial Laia, México, 3 edición, 1998

${ }^{2}$ La atención de los requerimientos sociales constituye una tendencia de la educación superior en Latinoamérica, siendo ilustrada para diferentes períodos por Luis Yarzabal. en: CARLOS TÜNNERMANN BERHEIM, La Educación Superior en el Umbral del Siglo XXI, Caracas, 1996, p. 5-6

${ }^{3}$ YARZABAL Luis., Op. cit., p. 6.

${ }^{4}$ ATCON, Rudolph. La universidad latinoamericana: clave para un enfoque conjunto del desarrollo coordinado social, económico y educativo en América Latina", en ECO, Tomo VII, Nos. 1-3, mayo-julio de 1993.

${ }^{5}$ CABALlERO Liliana (s.f.) Los fundamentos Constitucionales y Legales, en: Misión para la Modernización de la Universidad Pública, Estudios de Base, Tomo II, p. 303
} 
${ }^{6}$ El Plan de Apertura Educativa incorpora limitaciones a la política de financiación de la universidad estatal, planteando el "incremento progresivo de las matrículas en las universidades públicas de tal manera que los estudiantes cubran la tercera parte de los costos en un plazo de 10 años, creando sistemas de financiación para los estudiantes más pobres". Propone la participación de la banca con líneas de crédito para financiar los costos académicos y sostenimiento de estudiantes universitarios de familias de bajos ingresos. REPUBLICA DE COLOMBIA, Departamento Nacional de Planeación. Plan de Apertura Educativa 1991-1994- resumen ejecutivo, Bogotá, marzo 19 de 1991, Documento MEN-DNP- 2518- UDSDEC, p.14 y 20.

7 Al respecto de la cobertura de la educación superior se estima una "universidad de elite", cuando menos del 15\% de la cohorte (18-24 años) accede al nivel postsecundario; la "universidad de masas", oscila entre el 15\% y el 50\%, y la cobertura universitaria se transforma en "universal", cuando supera el 50\%. Verse: CABALLERO Liliana Op. Cit., p.302

${ }^{8}$ La ley 30 de 1992, establece en el Artículo 6 como uno de los objetivos de la educación superior "Promover la preservación de un medio amiente sano y fomentar la educación y cultura ecológica."

${ }^{9}$ MAZORRA Valderrama Miguel Ángel, los Alcances de la Investigación Ecológica, en: ECOBIOS Colombia 88 El desarrollo sostenible estrategias, políticas y Acciones, INDERENA, p.223-230

10 TAVERA Carmen. Legislación sobre Educación Ambiental, En: ECOBIOS Colombia 88 El desarrollo sostenible estrategias, políticas y Acciones, MINISTERIO DE AGRICULTURA, INDERENA, Bogotá, 1988, p.

${ }^{11}$ El Estado colombiano, fundamentado en la Ley 23 de 1973 estableció lineamientos de política para orientar la gestión ambiental, al igual que concedió facultades al Ejecutivo para expedir El Código Nacional de Recursos Naturales, Decreto 2811 de 1974, la reglamentación sobre la educación ambiental fue establecida posteriormente mediante la expedición del Decreto 1377 de julio 10 de 1978. El Código al respecto de la formación universitaria determino: "Dentro de las facultades que constitucionalmente le competen, el Gobierno al reglamentar la educación primaria, secundaria y universitaria, procurará: aIncluir cursos sobre ecología, preservación ambiental y recursos naturales renovables". REPÚBLICA DE COLOMBIA, Código Nacional de Recursos Naturales Renovables y de protección del Medio Ambiente, Decreto Número 2811 de diciembre 18 de 1974, Artículo 14.

12 Decreto 1377 de julio 10 de 1978, Artículo 7º.

${ }^{13}$ Decreto 1337 de julio 10 de 1978. El Articulo $8^{\circ}$ estableció: "La Comisión Asesora para la Educación Ecológica y del Ambiente", con funcionamiento en el Ministerio de Educación, conformada en el artículo $2^{\circ}$, a la que hace alusión este artículo, compuesta de la siguiente forma: Viceministro de educación o su delegado quien la presidirá; Director del ICFES o su delegado; Gerente del INDERENA o su delegado; Director General de capacitación, perfeccionamiento, currículo y medios educativos del MEN o su delegado; Subgerente del INDERENA o su delegado.

${ }^{14}$ ICFES. Contribución de la universidad al estudio y solución de los problemas ecológicos. Bogotá ICFES, Comité de Ecología, 1974.

${ }^{15}$ MAZORRA Valderrama, Op. Cit, p. 225-226

${ }^{16}$ El estudio del CIFCA sobre programas universitarios en el campo de las ciencias ambientales en Latinoamérica, se apoyo para el caso de Colombia, en el "Informe sobre Los Estudios Ambientales en Algunas de las Instituciones de Educación Superior en Colombia", elaborado por Oscar Marulanda y Humberto Rojas Ruiz, y alguna información las universidades de los Andes y Javeriana. CENTRO INTERNACIONAL DE FORMACIÓN EN CIENCIAS AMBIENTALES, La formación ambiental en América Latina, Madrid, CIFCA, 1978, Cuaderno \#8, p.60

${ }^{17}$ En este postgrado, el desarrollo regional, es examinado a través de las interrelaciones de los factores físicos, económicos, sociales y políticos, en este análisis intervienen especialistas en áreas de ingeniería, economía, administración, ciencia política, arquitectura, urbanismo y geografía El Plan de Estudios, incorpora 1- cursos de nivelación: Metodología científica, Introducción a la economía, conceptos y problemas de las ciencias sociales, matemáticas y estadística. 2- Cursos obligatorios: Teoría del desarrollo regional, Métodos de análisis regional, desarrollo regional en América Latina, Teoría de la planificación, Sistema de planificación en Colombia. 3- Cursos obligatorios: Metodología de la planificación regional, el Proceso del desarrollo regional en Colombia, cursos de especialización, planificación a nivel regional (urbano, rural, socioeconómico). Ibid., p.70 y p 101

${ }^{18}$ El estudio del CIFCA, determinó la existencia a finales de los años setenta de las siguientes tendencias: "Consideración del ambiente como un campo nuevo del conocimiento que requiere una teoría y una praxis tradicional; Aplicación de una metodología de enseñanza de carácter interdisciplinario; Superación 
de la actual compartimentación científica por disciplinas y establecimiento de nuevos mecanismos de relación interdepartamental o interfacultativa; Aplicación de enfoques integrados en proyectos específicos o trabajos de investigación, con la convergencia de profesionales de diversas disciplinas; Introducción de la dimensión ambiental, según diversas alternativas a nivel de grado y a nivel de postgrado. Ibid, p. 102.

19 Memorias del Primer Seminario Nacional sobre Universidad y Medio Ambiente.: Fondo José Celestino Mutis. Fondo Energético Nacional-FEN-INDERENA, COLCIENCIAS, Bogotá, Editora Guadalupe, 1989, p.20

${ }^{20}$ Declaración de Bogotá, publicada en: Memorias del Primer Seminario Nacional sobre Universidad y Medio Ambiente, 1988. Op. Cit p. 29-39.

21 Ángel (2000), Ob. Cit. p. 85.

${ }^{22}$ El balance de la incorporación de la dimensión ambiental en las funciones de la universidad colombiana en los años ochenta fue asumido en los siguientes estudios e informes: ÁNGEL MAYA, Augusto. Diagnóstico Nacional sobre Programas Ambientales Universitarios, Seminario Nacional sobre universidad y Medio Ambiente, ICFES, Universidad Nacional, Bogotá Abril 27 al 29 de 1988, Informe Final, Marzo de 1989; "Diagnóstico de la calidad de los estudios ambientales en Colombia" en 1990, POMBO, Diana y González, Edith, Eds. Perfil Ambiental de Colombia, AID, COLCIENCIAS, Fondo FEN, Bogotá, 1990. ICFES, BOHÓRQUEZ Camilo Antonio. Programas y actividades ambientales en la educación superior, Documento de trabajo, Subdirección Académica, Bogotá, abril de 1990

${ }^{23}$ ÁNGEL MAYA, Augusto. Diagnóstico Nacional sobre Programas Ambientales Universitarios, Seminario Nacional sobre universidad y Medio Ambiente, ICFES, Universidad Nacional, Bogotá Abril 27 al 29 de 1988, Informe Final, Marzo de 1989, Cáp. III, p.45.

${ }^{24}$ Ibid, Capitulo iii, p.1

${ }^{25}$ POMBO, Diana y González Edith (Eds.), op. Cit, p 280

${ }^{26}$ Estadísticas del Sistema de Información del ICFES, e informes de la Coordinación de la Red Nacional de Formación Ambiental. ICFES, SUBDIRECCIÓN ACADÉMICA, RED DE FORMACIÓN AMBIENTAL. Programas y actividades ambientales en la educación superior, Documento de trabajo, División de Formación Avanzada, Bogotá, 1990, p 15-16

${ }^{27}$ El Sistema de Ciencia y Tecnología fue configurado a partir de la expedición de la Ley de Ciencia y Tecnología, por el Congreso de la República (Ley 29 del 27 de febrero de 1990).

${ }^{28}$ El Decreto 585 de febrero 26 de 1991 considero como Programas de Ciencia y Tecnología: “un ámbito de preocupaciones científicas y tecnológicas estructurado por objetivos, metas y tareas fundamentales, que se materializan en proyectos y otras actividades complementarias que realizan entidades públicas o privadas, organizaciones comunitarias o personas naturales". Los programas de ciencia y tecnología adoptaron carácter nacional o regional. Fueron definidos como programas de ciencia y tecnología: a) el Programa de Ciencias Básicas; b) el Programa de Ciencias Sociales y Humanas; c) el Programa de Desarrollo Tecnológico Industrial y de Calidad; d) el Programa de Ciencia y tecnología agropecuarias; e) el Programa de Ciencias del Medio Ambiente y el Hábitat; f) el Programa de Estudios Científicos de la Educación; g) el Programa de Ciencia y tecnología de la Salud, H) los demás programas creados por el Consejo Nacional de Ciencia y tecnología. REPÚBLICA DE COLOMBIA, MINISTERIO DE GOBIERNO, DEPARTAMENTO NACIONAL DE PLANEACIÓN. Decreto número 585 del 26 de febrero de 1991, artículo 5.

${ }^{29}$ En 1992 se reunión en Villavicencio el Simposio Nacional del Programa Nacional de Estudios del Medio Ambiente y el Hábitat, con la participación de alrededor de 65 personas (investigadores, representantes gremiales, y administradores del sector). Definieron cinco líneas de investigación, el cambio climático global, diversidad biológica, desarrollo sostenible, asentamientos humanos, relación contaminación y tecnología. Al igual que la integración a redes internacionales y la creación de la Red de Investigadores Urbano-Regionales; la Red de Estudios Ambientales Urbanos; y la Red de Investigaciones sobre Biodiversidad. SÁENZ Orlando. Presentación del Programa Nacional de estudios del Medio Ambiente y del Hábitat. En COLCIENCIAS, El Entorno Nacional y Construido del Hombre colombiano, Bases para un Plan Del Programa Nacional de Ciencias del Medio Ambiente y el Hábitat, Bogotá, Tercer Mundo Editores,1993, p. X-XII.

${ }^{30}$ MÁRQUEZ Germán. Elementos para un Plan de investigaciones en Medio ambiente y hábitat, En: COLCIENCIAS, El Entorno Nacional y Construido del Hombre Colombiano, Bases para un Plan del Programa Nacional de Ciencias del Medio Ambiente y el Hábitat, Programas Nacionales en Ciencia y Tecnología. Coordinación Orlando Sáenz., Tercer Mundo Editores, Bogotá,1993 p. 30 
${ }^{31}$ La identificación de los programas de formación ambiental en 1999, reconoció la concepción utilizada en los estudios presentados en los Seminarios sobre Universidad y Medio Ambiente en América Latina y el Caribe en 1985, y el Primer Seminario Nacional en 1988. Los cuales asumen como programas de formación ambiental, aquellos cuyo objeto de estudio, comprende un espacio interdisciplinario que analiza la articulación sociedad- naturaleza.

32 En 1999 el Sistema de Información y Registro del ICFES, indicó la existencia de un total de 4015 programas en la educación superior, de los cuales 190 programas correspondían a formación ambiental en los niveles técnico tecnológico, profesional y posgraduado. Este conjunto de programas comprendía 40 programas en el campo de la ecología y 25 en el campo de medio ambiente y recursos naturales.

${ }^{33}$ HAJEK, R. Ernest, La incorporación de la dimensión ambiental en las ciencias naturales, En: Memorias del Seminario Universidad y medio Ambiente en América Latina y el Caribe, Op. Cit. p. 39.

34 ICFES-UNIVERSIDAD NACIONAL DE COLOMBIA I Seminario Nacional Sobre Universidad y Medio ambiente, Incorporación de la dimensión ambiental en la educación superior, Memorias del Seminario, Bogotá, 1989.p. 13

${ }^{35}$ La Ley 115 de 1994 y el Decreto 272 de 1998 determina que los programas de pregrado y postgrado para la formación de educadores corresponderían a niveles, modalidades y énfasis. La educación formal, comprendía tres niveles: preescolar, educación básica con dos ciclos educación básica primaria; y educación básica secundaria; y el nivel de educación media, con distintos énfasis. Las áreas obligatorias consideradas para la educación básica son: Ciencias naturales y educación Ambiental; Ciencias sociales; educación artística; educación ética y en valores humanos; educación física, recreación y deportes; educación religiosa; humanidades, lengua castellana e idiomas extranjeros; matemáticas; tecnología e informática.

\section{BIOGRAFÍA}

\section{MORELIA PABÓN PATIÑO}

Profesora Universidad Tecnológica de Pereira. Integrante del Grupo de Historia de la Universidad Colombiana, UTP, y del grupo HISULA. 